\begin{tabular}{|c|l|}
\hline Title & Formation mechanism of coarse columnar Y grains in as cast hy perperitectic carbon steels \\
\hline Author(s) & Tsuchiya, S.; Ohno, M.; Matsuura, K.; Isobe, K. \\
\hline Citation & $\begin{array}{l}\text { Acta Materialia, 59(9), 3334.3342 } \\
\text { https://doi.org/10.1016/.actamat.2011.02.007 }\end{array}$ \\
\hline Issue Date & 2011-05 \\
\hline Doc URL & http://hdl.handle.net/2115/45645 \\
\hline Type & article(author version) \\
\hline File Information & AM59-9_33343342.pdf \\
\hline
\end{tabular}

Instructions for use 


\title{
Formation mechanism of coarse columnar $\gamma$ grains in as-cast hyperperitectic carbon steels
}

\author{
Shingo Tsuchiya ${ }^{a}$, Munekazu Ohno ${ }^{\text {b, }{ }^{*} \text {, Kiyotaka Matsuura }}{ }^{b}$ and Kohichi Isobe ${ }^{\text {c }}$ \\ ${ }^{a}$ Graduate School of Engineering, Hokkaido University \\ ${ }^{\mathrm{b}}$ Division of Materials Science and Engineering, Faculty of Engineering, Hokkaido University, Kita \\ 13 Nishi 8, Kita-ku, Sapporo, Hokkaido, 060-8628, Japan \\ c Muroran R \& D lab., Nippon Steel Corp., 12 Nakamachi, Muroran, Hokkaido, 050-8550, Japan \\ * Corresponding author. Address: Division of Materials Science and Engineering, Faculty of
} Engineering, Hokkaido University, Kita 13 Nishi 8, Sapporo, Hokkaido, 060-8628, Japan. Tel/fax:

+81 11706 6344. E-mail address: mohno@eng.hokudai.ac.jp

\begin{abstract}
The formation mechanism of as-cast coarse columnar $\gamma$ grain (CCG) structure in hyperperitectic carbon steels is investigated by means of rapid unidirectional solidification method. This method realizes the cooling conditions similar to those in the vicinity of practical continuously cast slab surface. The microstructural observation of the quenched samples indicates that the CCG structure develops from the mold side along the direction of the temperature gradient. In the solidifying samples, fine columnar $\gamma$ grains (FCGs) always exist ahead of the CCG region. Instead of continuous growth into the large grains, the FCGs always shrink and vanish due to the growth of the CCGs initially formed near the mold side. Therefore, the grain size at a fixed point of the ingot discontinuously changes from the FCG to the CCG. The validity of this process was supported by numerical analyses. This finding is in marked contrast to the assumption made in the conventional grain growth analysis on the CCG structure.
\end{abstract}

Keywords: Casting; Grain growth; Columnar austenite grain; Grain size; Peritectic solidification 


\section{Introduction}

In continuous casting (C.C.) of peritectic carbon steels, as-cast $\gamma$-austenite structure consists of coarse columnar grains in the vicinity of the surface of the cast steel slab. The coarse columnar $\gamma$ grain (CCG) structure causes the detrimental effects on the ductility of the slab and causes surface cracking in C.C. and/or direct hot rolling processes $[1,2]$. Hence, it is quite important to prevent the formation of the CCG structure.

The solidification of the peritectic carbon steel starts with the crystallization of primary $\delta$-ferrite phase, followed by the peritectic reaction, $\mathrm{L}+\delta \rightarrow \gamma$, at the peritectic reaction temperature, $T_{\mathrm{P}}$. The $\gamma$ phase is coexistent with the liquid or $\delta$ phase in a temperature range between $T_{\mathrm{P}}$ and a temperature for completion of $\gamma$ transformation, $T_{\gamma}$. In this range, the liquid or $\delta$ phase acts as a pinning phase for $\gamma$ grain growth [2-4]. The grain growth of the $\gamma$ phase takes place immediately after the liquid or $\delta$ phase disappears below $T_{\gamma}[2-4]$. This behavior was well substantiated for the formation of equiaxed $\gamma$ grain structure in the slow cooling process with small temperature gradient [2-4].

The formation of as-cast CCG structure in slow cooling process with a large temperature gradient was investigated for the hyperperitectic carbon steel by means of 
Bridgman type unidirectional solidification method [5]. It was observed that the CCG structure develops along the temperature gradient. Importantly, it was found that fine columnar $\gamma$ grains (FCGs) always form ahead of the CCG region. The temperature in the FCG region corresponds to the liquid $+\gamma$ two phase field in the phase diagram. The minor axis diameter of the FCG is comparable to the primary dendrite arm spacing [5].

Based on these experimental findings [2,5], a classical grain growth model was applied to the prediction of grain size in as-cast columnar $\gamma$ grain structure [6]. The assumption on which this model is based is schematically illustrated for the case of hyperperitectic carbon steel in Fig. 1. When one considers the position denoted by " $H$ ", this region corresponds to the $\mathrm{L}+\gamma$ two phase field in Fig. 1(a). The liquid phase surrounds the $\gamma$ grains, accordingly retarding the growth of $\gamma$ grains. These $\gamma$ grains correspond to the FCGs. When the temperature at " $H$ " becomes lower than $T_{\gamma}$ (Fig. 1(b)), the FCG is expected to continuously grow and finally become the CCG (Fig. 1(c)). Hence, this process assumes that the grain growth continuously takes place from the FCG to CCG in the cooling process below $T_{\gamma}$. It has been demonstrated that the classical model based on this assumption is useful in predicting the $\gamma$ grain size in some C.C. slabs cooled down to a low temperature [6-9]. To the best of the present authors' knowledge, however, there is no direct experimental evidence indicating the continuous 
grain growth from the FCG to CCG. Therefore, despite its practical importance and scientific interest, the formation mechanism of the CCG structure remains to be clarified.

In the present study, we carry out the detailed investigation on the formation mechanism of the CCG structure in a rapid cooling process with a large temperature gradient. In particular, our focus is directed to the cooling process similar to that in the slab surface in the practical C.C. process. For this, we employ rapid unidirectional solidification method that enables us to examine the as-cast $\gamma$ grain structure quite similar to that observed in the practical C.C. slab surface. We focus on a peritectic carbon steel with $0.2 \mathrm{mass} \%$ carbon concentration, since the as-cast $\gamma$ grain structure in this steel is known to exhibit quite coarse and columnar structure [10]. Furthermore, we investigate 0.35 mass $\%$ carbon steel in order to support the validity of our findings.

In this paper, we demonstrate that the continuous growth from the FCG to CCG does not occur but the change from the FCG to CCG structure is discontinuous. The CCGs continuously develop from the mold side along the temperature gradient. Although the FCGs always form ahead of the CCGs, the FCGs do not grow to be the CCGs. The FCGs always shrink due to the growth of the CCGs. Moreover, the coarsening of the CCG along the minor axis diameter occurs only at temperatures close 
to $T_{\gamma}$, and the grain growth barely takes place below $T_{\gamma}$. These findings are in marked contrast to the assumption (Fig. 1) in the previous studies [6-8] that the CCG structure results from the continuous growth from the FCGs during the cooling process below $T_{\gamma}$.

\section{Experimental}

In the present study, we focus on the hyperperitectic carbon steels with 0.2 and 0.35 mass $\%$ carbon concentrations. These steels are called $0.2 \mathrm{C}$ and $0.35 \mathrm{C}$ in this paper and their chemical compositions are shown in Table 1. A forged 0.2 mass $\%$ carbon steel bar was utilized as a row material. The $0.35 \mathrm{C}$ steel sample was prepared with mixing the forged 0.2 mass $\%$ steel bar and a forged 0.45 mass $\%$ carbon steel bar. The samples were melted in a cylindrical $\mathrm{MgO}$ crucible with an inner diameter of $28 \mathrm{~mm}$ and a depth of $70 \mathrm{~mm}$ at $1570^{\circ} \mathrm{C}$ in a $\mathrm{SiC}$ furnace filled with the Ar gas of five-nine purity and held at this temperature for an hour. Then, the melted sample was cast into the rapid unidirectional solidification equipment. The cast sample was about $180 \mathrm{~g}$ in weight and $40 \mathrm{~mm}$ in height. Figure 2(a) is the schematic illustration of this equipment. The melted sample was cast into an $\mathrm{MgO}$ pipe on a water-cooled copper mold. The $\mathrm{MgO}$ pipe had been preheated at $1570^{\circ} \mathrm{C}$ and put on the copper mold just before the casting operation to realize upward unidirectional solidification. 
In order to check the cooling condition in this equipment, we preliminarily monitored the cooling curves at different positions in the samples by setting B-type thermocouples inside the sample at several heights from the copper mold. Figure 2(b) shows the cooling curves at 10 and $15 \mathrm{~mm}$ heights for the $0.2 \mathrm{C}$ sample. A dashed line in Fig. 2(b) indicates $T_{\gamma}$ for the $0.2 \mathrm{C}$ sample calculated by the CALPHAD method [11]. The thermodynamic database, PanIron [12], was employed for this calculation. The values of $T_{\gamma}$ were calculated to be $T_{\gamma}=1478{ }^{\circ} \mathrm{C}$ for the $0.2 \mathrm{C}$ sample and $T_{\gamma}=1448^{\circ} \mathrm{C}$ for the $0.35 \mathrm{C}$ sample. Both the cooling rates at 10 and $15 \mathrm{~mm}$ heights were estimated to be about $10^{\circ} \mathrm{C} / \mathrm{s}$ at $T_{\gamma}$. This value is in good agreement with the cooling rate estimated for the practical C.C. process [13]. The temporal change of the microstructure in the solidifying sample was investigated by dropping the solidifying sample together with the $\mathrm{MgO}$ pipe quickly into intensely agitated iced water. The quenching equipment consists of the water vessel and the rotation agitator with chains at the center of water vessel. The agitator with chain enables us to realize rapid cooling of the solidified samples by crashing the $\mathrm{MgO}$ pipe. As mentioned later, in this rapid quenching technique, almost all the liquid phases remaining in the sample were dropped out of the sample, leaving only the solidified sample after the quenching operation. The solidification was completed over the entire sample after holding for $100 \mathrm{~s}$ in the 
equipment. The time for quenching operation was accordingly chosen to be $10-100$ s.

Each ingot was sectioned vertically along the center axis of the ingot and the cross sections were polished and etched with 3\%-nital and Oberhoffer's solutions to observe the as-cast $\gamma$ structure and the dendrite structure, respectively. For the microstructural observation, we focused on the region from the bottom to $25 \mathrm{~mm}$ height from the mold because the cooling process at the top part of the sample should be affected by the air cooling condition which is not our concern. Also, the sample was horizontally sectioned at several heights from the copper mold wall and the minor axis diameter of the $\gamma$ grains was measured on the horizontal sections.

\section{Results and discussion}

\subsection{Microstructures in the samples cooled to room temperature}

Figures 3(a) and (b) show the as-cast $\gamma$ structure and dendrite structure, respectively, in the same region of the $0.2 \mathrm{C}$ sample. The sample was cooled down to room temperature (RT) in the equipment without quenching. It is seen that the CCGs develop from the mold side toward the upper side. In Fig. 3(b), the columnar dendrites unidirectionally develop from the mold side toward the upper side of the sample. The similar structures were also observed in the $0.35 \mathrm{C}$ sample. From these microstructural 
observations, we can confirm that the unidirectional solidification was realized over the whole observation area.

Figure 4 shows the variation of the minor axis diameter of $C C G, D_{\mathrm{CCG}}$, in the $0.2 \mathrm{C}$ and $0.35 \mathrm{C}$ samples naturally cooled to RT. The horizontal axis is the distance from the mold wall (or height from the mold wall). In each sample, $D_{\mathrm{CCG}}$ increases as the distance from the mold wall increases. For comparison, the data obtained from the C.C. slabs [13] are plotted in Fig. 4. The data in Ref. [13] originally indicate average area per $\gamma$ grain on the section parallel to the side surface. We converted these data to the average diameter by assuming the circular cylindrical shaped grains. $C p$ in Fig. 4 indicates the equivalent carbon content of the steels. This value was calculated by using the equation proposed by Howe [14], $C p=C_{C}-0.14 C_{S i}+0.04 C_{M n}$, with the concentration of $i$ element, $C_{i}(\operatorname{mass} \%)$. The values of $D_{\mathrm{CCG}}$ in the C.C. slabs with $C p$ $=0.21$ and 0.19 mass $\%$ [13] are slightly higher than those in the $0.2 \mathrm{C}$ sample. However, the difference is not substantial at all. One can grasp that the present casting process yields the as-cast $\gamma$ grain structure quite similar to that observed in the practical C.C. process. In Fig. 4 , the values for the $0.35 \mathrm{C}$ sample are always lower than those for the $0.2 \mathrm{C}$ sample, which originates from the difference in $T_{\gamma}$ between these samples as discussed later. 


\subsection{Microstructures in solidifying samples}

Figures 5(a) and (b) show the as-cast $\gamma$ grain structures of the $0.2 \mathrm{C}$ samples quenched at $30 \mathrm{~s}$ and $50 \mathrm{~s}$, respectively, after the casting operation. The total height of the sample quenched at $30 \mathrm{~s}$ is lower than that quenched at $50 \mathrm{~s}$. This is because the liquid metal was dropped out of the sample during quenching operation in intensely agitated iced water as already mentioned in the section 2 . From the microstructural observation, it was found that the additional solidification of the remaining liquid on the top part of the already solidified sample did not occur during the present quenching operation. The CCG region gradually develops from the mold side toward the upper side of the sample. Importantly, the very fine columnar grains, viz., FCGs exist ahead of the CCG region in Figs. 5(a) and (b). The formation of the FCGs was observed in all the samples quenched at different timings and also in all the $0.35 \mathrm{C}$ samples. The minor axis diameter of FCG is almost an order of magnitude less than that of CCG. Figure 5(c) shows the $\gamma$ grain structure in the vicinity of the boundary between the FCG and $\mathrm{CCG}$ regions (FCG/CCG region boundary) in the $0.2 \mathrm{C}$ sample quenched at $30 \mathrm{~s}$. In Fig. 5(c), the $\gamma$ grain structure abruptly changes from the FCG to CCG. This abrupt change of the $\gamma$ grain structure was observed in all the solidifying samples. Figure 5(d) shows 
the dendrite structure in the same region as in Fig. 5(c). The columnar dendrites continuously develop from the bottom to the top part of the figure and there is no abrupt change in the dendrite structure at the FCG/CCG region boundary. From the detailed comparison between Figs. 5(c) and (d), it was found that the position of the $\gamma$ grain boundary in FCG region mostly corresponds to the interdendritic position. The minor axis diameter of FCG is approximately equivalent to the primary dendrite arm spacing $(\sim 100 \mu \mathrm{m})$. These facts were also observed in the $0.35 \mathrm{C}$ samples. The FCG structure observed in this study is quite similar to the structure observed in the slow cooled sample in the Bridgman type unidirectional solidification equipment [5].

The microstructural observation of the solidifying samples showed that the FCG/CCG region boundary gradually moves from the copper mold side to the upper part of the ingot. We measured the migration distance of the FCG/CCG region boundary from the mold wall, $H_{\mathrm{CCG}}$. The time variations of $H_{\mathrm{CCG}}$ in the $0.2 \mathrm{C}$ and $0.35 \mathrm{C}$ samples are shown in Fig. 6 . In both the samples, $H_{\mathrm{CCG}}$ gradually increases with the time. The open marks in Fig. 6 indicate the time when the temperature reaches $T_{\gamma}$ in the cooling curve monitored at each position. It is very important to note that the open marks are located almost on the curve for $H_{\mathrm{CCG}}$ in each sample. This fact indicates that the temperature at the FCG/CCG region boundary is always equal to $T_{\gamma}$ during the 
solidification in both the samples. Therefore, the FCG region corresponds to the liquid $+\gamma$ two phase field. In the early study on slow cooling process [5], the FCG region was also found to correspond to the liquid $+\gamma$ two phase field. Also, it was concluded that the liquid phase in the FCG region acts as the pining phase for the $\gamma$ grain growth $[5]$

As shown in Fig. 5(c), the structure discontinuously changes from the FCG to the CCG. This is in marked contrast to the assumption in the early studies that the FCGs continuously grow and become the CCGs [6-8]. This point is further addressed in the next section.

\section{3. Grain growth along minor axis of columnar grains}

As mentioned, the early studies on the formation of CCG structure [6-8] assumed that the FCGs continuously grow into the CCGs during the cooling process below $T_{\gamma}$. However, as shown in Fig. 5(c), the $\gamma$ grain structure discontinuously changes from the FCG to CCG at the FCG/CCG region boundary. Therefore, in order to check the validity of the conventional assumption, we performed the following measurement. We measured the minor axis diameter of $\gamma$ grains at a fixed position (a fixed height from the mold wall) in the $0.2 \mathrm{C}$ samples quenched at different timings. We chose $8 \mathrm{~mm}$ 
height from the mold wall. Then, we converted the quenching timing to the temperature based on the measured cooling curve at $8 \mathrm{~mm}$ height. The results are shown by the filled square symbols in Fig. 7. As indicated by the arrows, the diameter discontinuously and drastically increases by almost one order of magnitude at $T_{\gamma}$. Furthermore, the diameter does not appreciably change below $T_{\gamma}$.

For comparison, we carried out the calculation for the grain growth based on the conventional assumption that the FCG continuously grows into the CCG during the cooling process below $T_{\gamma}$. The grain growth model based on the curvature driven growth is expressed by the following equation [15],

$$
\frac{d D}{d t}=M \cdot \frac{1}{D}=M_{0} \exp \left(-\frac{Q}{R T}\right) \cdot \frac{1}{D}
$$

where $D$ is average grain diameter $(\mathrm{m}), M$ is the grain boundary mobility $\left(\mathrm{m}^{2} \cdot \mathrm{s}^{-1}\right), M_{0}$ represents the kinetic constant for the grain boundary mobility $\left(\mathrm{m}^{2} \cdot \mathrm{s}^{-1}\right)$ and $Q$ is the apparent activation energy for grain growth $(\mathrm{kJ} / \mathrm{mol})$. In Ref. [16], the values of $Q$ and $M_{0}$ were measured for the three-dimensional grain growth in the slow cooling process of plain carbon steels. Our concern here is the two-dimensional grain growth of the minor axis diameter of the columnar $\gamma$ grains. For the present calculation of the $0.2 \mathrm{C}$ sample, we employed $Q=177 \mathrm{~kJ} / \mathrm{mol}$ measured in Ref. [16] and $M_{0}=2.0 \times 10^{-3} \mathrm{~m}^{2} \cdot \mathrm{s}^{-1}$ which is one-half of the value obtained in Ref. [16]. On the basis of the curvature driven grain 
growth theory, the driving force for the two-dimensional grain growth must be half of that of the three-dimensional grain growth [17]. For the numerical analysis, by taking this fact into account, we used one-half of the value reported in Ref. [16] for $\mathrm{M}_{0}$.

The equation (1) was solved based on the finite difference method. We employed the experimentally obtained cooling curve for the time variation of temperature in eq. (1). The calculated result is indicated by the dashed line in Fig. 7. One can see that the continuous grain growth model considerably underestimates the minor axis diameter of the $\gamma$ grains. However, there might be a possibility that the above mentioned input parameters are not appropriate for the present case. Hence, we tested the different value of $M_{0}$. The dotted line represents the result calculated with $M_{0}=0.014 \mathrm{~m}^{2} \cdot \mathrm{s}^{-1}$. This value was obtained so as to reproduce the experimental data in the low temperature region. From this result, it is evident that the drastic change of the diameter near $T_{\gamma}$ cannot be explained by the continuous grain growth model. Therefore, it is revealed that the continuous grain growth from the FCG to CCG below $T_{\gamma}$ does not take place. We confirmed that this is the case at the different position in the $0.2 \mathrm{C}$ sample and also in the $0.35 \mathrm{C}$ sample. Therefore, the formation mechanism of the CCG structure should be different from the previous model [6-8].

As shown in Fig. 7, the minor axis diameter does not change below $T_{\gamma}$. On the 
other hand, the minor axis diameter gradually increases with increasing distance from the mold wall in the samples naturally cooled to RT (Fig. 4). In order to clarify this behavior, we measured $D_{\mathrm{CCG}}$ at the FCG/CCG region boundary in the $0.2 \mathrm{C}$ samples quenched at the different timings. The result is shown by the filled square symbols in Fig. 8 . One can see that $D_{\mathrm{CCG}}$ at the FCG/CCG region boundary gradually increases with the time after casting. Although not shown here, the value of $D_{\mathrm{CCG}}$ in Fig. 8 is almost coincident with the value of $D_{\mathrm{CCG}}$ at the corresponding position in the sample naturally cooled to RT (Fig. 4). This fact indicates that the minor axis diameter increases only in the vicinity of the growing tip of CCG region where $T=T_{\gamma}$ and it does not change below $T_{\gamma}$. This is also the case in the $0.35 \mathrm{C}$ sample.

It is noted that the temperature at the FCG/CCG region boundary always corresponds to $T_{\gamma}$ during the solidification (Fig. 6). Hence, the increase of $D_{\mathrm{CCG}}$ at the growing tip of CCG region (FCG/CCG region boundary) in Fig. 8 may be explained by the isothermal grain growth process near $T_{\gamma}$. We calculated this isothermal process based on eq. (1). The calculated results are shown in Fig. 8. The initial diameter was assumed to be $D_{0}=0.46 \mathrm{~mm}$. This value corresponds to $D_{\mathrm{CCG}}$ at $1 \mathrm{~mm}$ away from the mold wall in the sample naturally cooled to RT. We found it necessary to modify the value of $M$ reported in Ref. [16] in order to describe the present experimental data of 
the $0.2 \mathrm{C}$ and $0.35 \mathrm{C}$ samples. Assuming $Q=177 \mathrm{~kJ} / \mathrm{mol}[16]$, we estimated the appropriate value of $M_{0}$ for the present case to be $0.013 \mathrm{~m}^{2} \cdot \mathrm{s}^{-1}$. The calculated result for the $0.2 \mathrm{C}$ sample is indicated by the solid line in Fig. 8 . The isothermal grain growth model reproduces the experimental data very well. This indicates that the minor axis diameter of the CCGs increases in the isothermal grain growth at $T_{\gamma}$. Hence, the growth of the CCG along the minor axis diameter occurs only in the vicinity of the growing tip of the CCG region, where $T \approx T_{\gamma}$. Once the $\gamma$ grains become coarse near $T_{\gamma}$, the driving force for the subsequent grain growth below $T_{\gamma}$ almost vanishes and the diameter does not change below $T_{\gamma}$.

It is noted that the present calculation for the isothermal grain growth at $T_{\gamma}$ can also reproduce the time dependence of $D_{\mathrm{CCG}}$ for the $0.35 \mathrm{C}$ sample. Since $T_{\gamma}$ for the $0.35 \mathrm{C}$ sample is lower than the one for the $0.2 \mathrm{C}$ sample, $D_{\mathrm{CCG}}$ for the $0.35 \mathrm{C}$ sample is always smaller than that for the $0.2 \mathrm{C}$ sample in each time period. As a result, $D_{\mathrm{CCG}}$ for the $0.35 \mathrm{C}$ sample naturally cooled to RT is always smaller than that for the $0.2 \mathrm{C}$ sample as seen in Fig. 4.

In the above-mentioned calculation, the value of $M_{0}$ is significantly higher than the value reported in Ref. [16]. Instead of changing $M_{0}$, we may modify the value of $Q$ to reproduce the present experimental data. There are several possible reasons to 
explain the difference in $M_{0}$ and/or $Q$ between the present work and Ref. [16] such as the difference in the temperature range for the estimation of these values and the possibility of the concentration dependence of these values [18]. However, the detailed discussion about this point is beyond the focus of this study. For the present purpose, it is sufficient to choose the values of $M_{0}$ and $Q$ to judge whether or not the grain growth observed in the $0.2 \mathrm{C}$ and $0.35 \mathrm{C}$ samples can be reproduced by the model based on the curvature driven growth, eq(1).

\subsection{Formation mechanism of coarse columnar $\gamma$ structure}

The present investigation demonstrated the following facts.

1) The FCGs always exist ahead of the CCG region (Fig. 5).

2) The temperature at the FCG/CCG region boundary is always near $T_{\gamma}$ during the solidification (Fig. 6). The FCG region thereby corresponds to the liquid $+\gamma$ two phase field.

3) The minor axis diameter of the FCG corresponds to the primary dendrite arm spacing $(\sim 100 \mu \mathrm{m})$. The positions of grain boundaries in the FCG region are mostly in accordance with the position of interdendritic positions of the columnar dendrites. 
4) The minor axis diameter of the columnar $\gamma$ grains at a fixed point of the ingot discontinuously changes from the one of FCG to CCG. The grain growth along the minor axis does not take place below $T_{\gamma}($ Fig. 7).

5) The grain growth along the minor axis of CCG isothermally takes place at $T_{\gamma}$ only near the FCG/CCG region boundary (Fig. 8).

From these findings, we consider the following scenario for the formation process of the CCG structure, as schematically shown in Fig. 9(a). First, the columnar dendrite of $\delta$-ferrite phase develops from the mold wall along the temperature gradient. Near the mold side, $\gamma$ phase forms when the temperature is lower than the peritectic temperature, $T_{\mathrm{P}}$, and then the $\gamma$ grains coarsen below $T_{\gamma}$ as shown in Fig. 9(a)-(i), although this initial formation process of the CCG is not clear in the present study. Ahead of the CCGs, the new $\gamma$ grains form from the $\delta$-ferrite dendrite below $T_{\mathrm{P}}$ and they grow to become the FCGs. In this region (FCG region), the liquid phase pins the growth of FCG along its minor axis direction. This liquid phase also pins the upward growth of the CCGs. When the temperature in the FCG region becomes lower than $T_{\gamma}$, the CCGs grow upward and the FCG shrinks as shown in Figs. 9(a)-(ii) and 9(a)-(iii). The grain growth along the minor axis of CCG isothermally takes place at $T \approx T_{\gamma}$ only near the FCG/CCG region boundary. 
As demonstrated above, the FCG/CCG region boundary always corresponds to $T_{\gamma}$ and, therefore, the motion of the FCG/CCG region boundary is considered to be pinned by the liquid phase. If there is no pinning effect of the liquid phase, the growth velocity of the $\mathrm{CCG}$ along its major axis direction, $V_{\mathrm{CCG}}$, should be entirely determined by the conventional curvature driven growth at $T_{\gamma}$. In order for the proposed process shown in Fig. 9(a) to be realized, $V_{\mathrm{CCG}}$ should be higher than or be comparable to the measured velocity in Fig. 6, $V_{\gamma}$. Hence, we estimate $V_{\mathrm{CCG}}$ and compare it with $V_{\gamma}$ to check the validity of our proposed process.

The minor axis diameter of the FCG is almost an order of magnitude smaller than that of the CCG. The driving force for the migration of FCG/CCG region boundary should be ascribable to the minimization of the total grain boundary energy. Hence, the large difference in the minor axis diameter yields the driving force for the migration of FCG/CCG region boundary. The FCGs neighboring the CCGs should shrink as indicated by the arrow in Fig. 9(a)-(ii). Hence, $V_{\mathrm{CCG}}$ corresponds to the shrinking velocity of the FCG. We estimate the shrinking velocity of the FCG based on the curvature driven growth. We consider three-dimensional curvature radius of FCG at the FCG/CCG region boundary, $r$, as shown in Fig. 9(b). Based on eq. (1), then, $V_{\mathrm{CCG}}$ can be calculated from the following equation, 


$$
V_{\mathrm{CCG}}=\frac{d r}{d t}=\frac{1}{4} \cdot 2 M_{0} \exp \left(\frac{-Q}{R T}\right) \cdot \frac{1}{r}
$$

As for the value of $M_{0}$, we employed the value of $M_{0}=0.013 \mathrm{~m}^{2} \cdot \mathrm{s}^{-1}$. Then, $V_{\mathrm{CCG}}$ at $T_{\gamma}(0.2 \mathrm{C})=1478^{\circ} \mathrm{C}$ was calculated to be 0.45 and $1.36 \mathrm{~mm} / \mathrm{s}$ for $r=150$ and $50 \mu \mathrm{m}$, respectively. Also, $V_{\mathrm{CCG}}$ at $T_{\gamma}(0.35 \mathrm{C})=1448^{\circ} \mathrm{C}$ was calculated to be 0.36 and $1.05 \mathrm{~mm} / \mathrm{s}$ for $r=150$ and $50 \mu \mathrm{m}$, respectively. These values are quite comparable to or higher than $V_{\gamma}=0.27-0.45 \mathrm{~mm} / \mathrm{s}$ for both $0.2 \mathrm{C}$ and $0.35 \mathrm{C}$ samples estimated from Fig. 6 . Therefore, the FCG/CCG region boundary can migrate at a rate higher $V_{\gamma}$ if the pinning of the liquid phase does not exist. This analysis strongly supports the validity of the scenario shown in Fig. 9(a).

It is noted that the scenario of the CCG formation process proposed here is essentially based on the pinning effect of liquid phase on FCG/CCG region boundary. As described in the introduction, the early literatures [2-4] demonstrated that the liquid phase acts as a pinning phase in the $\gamma$ grain growth. As is commonly discussed within the framework of Zener pinning [19], when the $\gamma$ grain boundary intersects a liquid phase, the total energy of $\gamma$ grain boundary is reduced. Therefore, the unpinning process generally requires increment of the total grain boundary energy, which leads to the pinning force, $F_{d}$. The Zener pinning pressure $P_{z}$ can be then calculated to be $P_{z}=F_{d} \cdot n_{d}$ 
where $n_{d}$ is the number density of the pinning (liquid) phase per unit area of the $\gamma$ grain boundary. The value of $F_{d}$ depends substantially on the size and the shape of the pinning phase as discussed in detail by Nes et al.[20]. In the present case, hence, the value of $F_{d}$ is mainly determined by the shape and size of the solidifying liquid phases, which depends on the detail of the dendrite structure. In the present case, furthermore, the distribution of the pinning phases (liquid phases) is not uniform but depends on the local position in the dendrite structure formed during unidirectional solidification. Hence, the estimation of $F_{d}$ and $n_{d}$ requires the detailed analysis on the shape and size of solidifying liquid phases in the three dimensional dendrite structure, which is a challenging task and is beyond our concern in this paper.

Since the volume fraction of liquid phase increases with the temperature in liquid $+\gamma$ phase field, the size and the number of the pinning phases (liquid phases) during the unidirectional solidification process generally increase with the increase in the local temperature at which the FCG/CCG region boundary exists. The pinning pressure then increases as the FCG/CCG region boundary migrates toward the high temperature region due to the increases in $F_{d}$ and $n_{d}$. Hence, if the FCG/CCG region boundary overcomes the pinning pressure of the liquid phases at a certain position, the FCG/CCG boundary is again pinned by higher pinning pressure in the higher 
temperature region. Therefore, one can expect that the FCG/CCG region boundary should be necessarily pinned in liquid $+\gamma$ phase region or the FCG region during the unidirectional solidification process. This pinning effect yields the discontinuous nature of FCG/CCG transition. The detailed analysis on this pinning effect requires the analysis on the three-dimensional dendritic structure and the microsegregation patterns during unidirectional solidification process which remains as a future work.

\section{Conclusions}

In the present study, the formation kinetics of coarse columnar $\gamma$ grain $(\mathrm{CCG})$ structure in hyperperitectic carbon steels was investigated by using the rapid unidirectional solidification equipment. It was found that the formation process of the CCG structure is different from the conventional assumption for the continuous grain growth from fine columnar $\gamma$ grain (FCG). The important findings in the present study are summarized as follows:

(1) The FCGs always exist ahead of the CCG region during the solidification. The change from the FCG to CCG structure is discontinuous. The minor axis diameter of FCG is quite comparable to the primary dendrite arm spacing and the FCG region corresponds to the liquid $+\gamma$ two phase field. 
(2) The migration distance of the FCG/CCG region boundary gradually increases with time. The CCG region develops toward the FCG region when the temperature of the FCG/CCG region boundary becomes lower than $T_{\gamma}$. The driving force for the migration of FCG/CCG region boundary should be ascribable to the minimization of the total grain boundary energy.

(3) The grain growth along the minor axis of CCG isothermally takes place at $T \approx T_{\gamma}$ only near the FCG/CCG region boundary. The CCG does not coarsen below $T_{\gamma}$.

\section{Acknowledgement}

This work is partly supported by $18^{\text {th }}$ ISIJ Research Promotion Grant and Grant-in-Aid for Young Scientists (a) (No. 22686067) from MEXT, Japan.

\section{References}

[1] Schmidt L and Josefsson Å. Scand J Met 1974;3:193.

[2] Maehara Y, Yasumoto K, Sugitani Y, Gunji K. ISIJ Int 1985;25:1045.

[3] Pottore NS, Garcia CI and DeArdo AJ. Metall Trans A 1991;22A:1871.

[4] Tsuchiya S, Ohno M and Matsuura K. Tetsu-to-Hagané 2009;95:629.

[5] Maruyama T, Matsuura K, Kudoh M and Itoh Y. Tetsu-to-Hagané 1999;85:585.

[6] Yoshida N, Umezawa O and Nagai K. ISIJ Int 2004;44:547.

[7] Yoshida N, Kobayashi Y and Nagai K. Tetsu-to-Hagané 2004;90:198.

[8] Kobayashi Y, Iwasaki S, Nakazato K, Hibaru T, Kuroda S, Sakuma N, Yoshida N and Nagai K. ISIJ Int 2008;48:344.

[9] Kim HS, Kobayashi Y and Nagai K. Acta Metall 2006;54:2441.

[10] Ohba Y, Kitade S and Takasu I. ISIJ Int 2008;48:350.

[11] Kaufman L and Bernstein L. Computer Calculation of Phase Diagrams with Special Reference to Refractory Materials. Academic Press. New York. 1970. 1. [12] CompuTherm LLC: http://www.computherm.com/. Last accessed date: 08/09/2010.

[13] Reiter J, Bernhard C and Presslinger H. Mater Charact 2008;59:737. 
[14] Howe AA. Segregation and Phase Distribution During Solidification of Carbon, Alloy and Stainless Steels. European Communities / Union (EUR-OP/OOPEC/OPOCE). Luxemburg. 1991. pp.31-40.

[15] Andersen I and Grong Ø. Acta Metall 1995;43:2673.

[16] Schwerdtfeger K, Köthe A, Rodriguez JM and Bleck W. Thin Slab Casting. EUR 19409/1 EN. Luxembourg. 2001. vol. 1, pp.33-48.

[17] Hillert M. Acta Metall 1965;19;227.

[18] Bernhard C, Reiter J and Presslinger H. Metall Trans B 2008;39B:885.

[19] Manohar PA, Ferry M and Chandra T. ISIJ Int 1998; 38; 913.

[20] Nes E, Ryum N and Hunderi O. Acta metal 1985; 33; 11. 
Table 1. Chemical compositions of the samples employed in this study.

Sample

Steel composition, in Mass \%

\begin{tabular}{ccccccccc} 
name & C & Si & Mn & P & S & Al & O & N \\
\hline 0.2C & 0.20 & 0.21 & 0.79 & 0.02 & 0.003 & 0.04 & 0.0027 & 0.0051 \\
$0.35 C$ & 0.35 & 0.21 & 0.74 & 0.02 & 0.01 & 0.03 & 0.002 & 0.0053
\end{tabular}


(a)

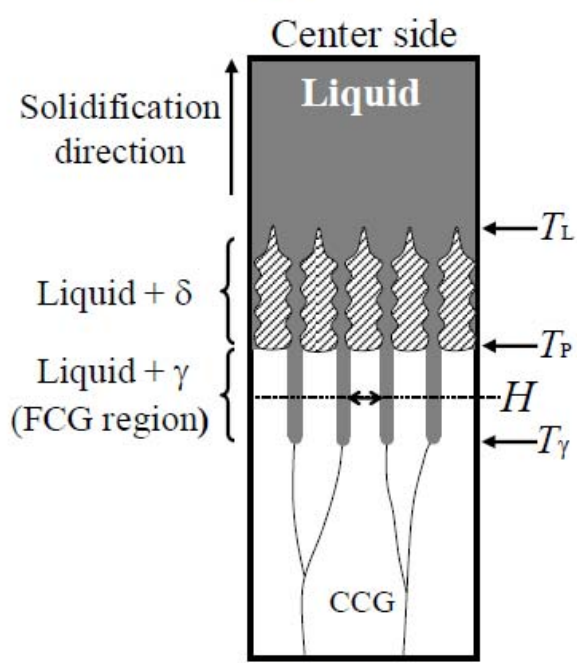

Mold side (b)

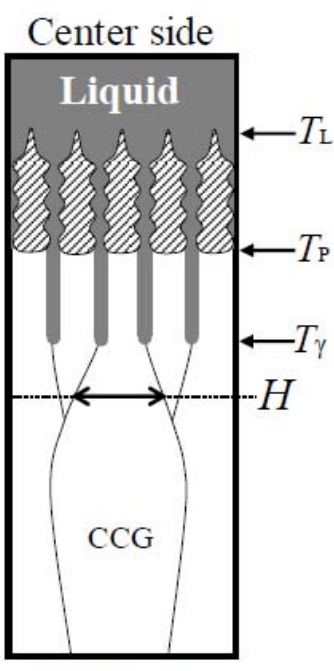

Mold side (c)

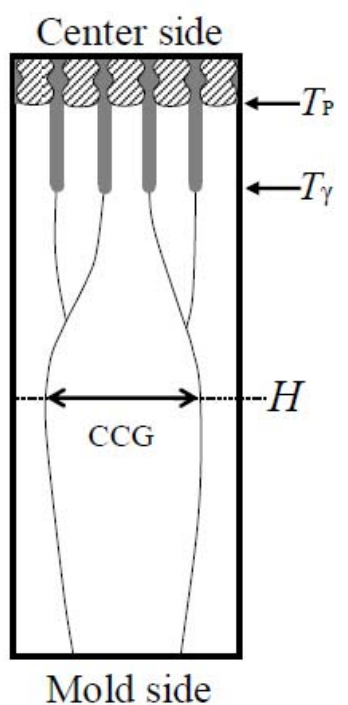

$T_{\mathrm{L}}$ Liquidus temperature

$T_{\mathrm{P}}$ Peritectic temperature

$T_{\gamma}$ Temperature for completion of $\gamma$ transformation

Fig. 1. Schematic illustration of the formation process of the CCG structure assumed in

Ref. [6]. The solidification proceeds from (a) to (c).

Fig. 1. Schematic illustration of the formation process of the CCG structure assumed in

Ref. [6]. The solidification proceeds from (a) to (c). 
(a)

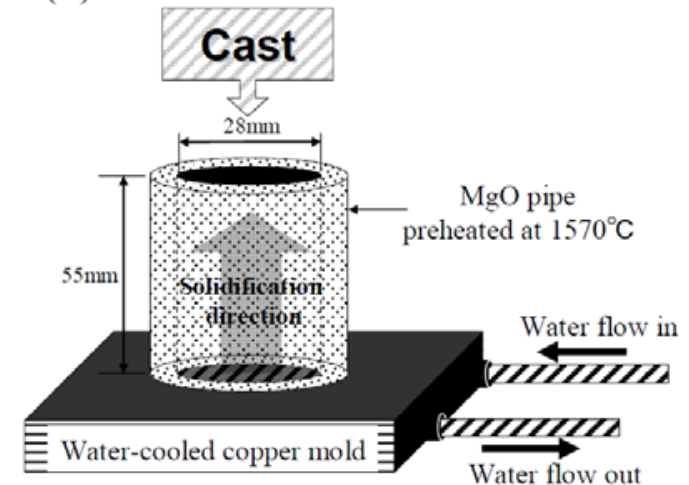

(b)

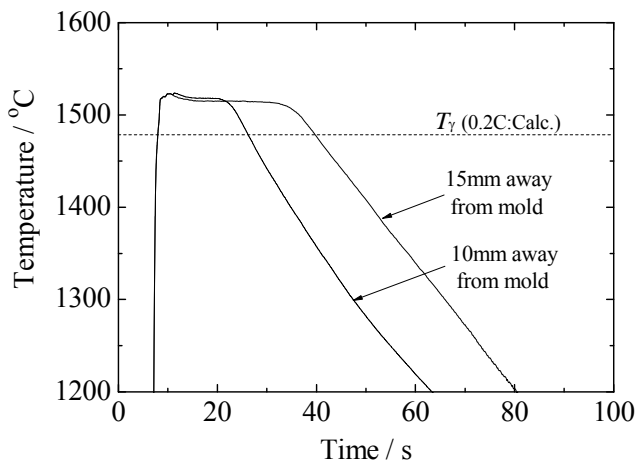

Fig. 2. (a) Schematic illustration of the rapid unidirectional solidification equipment used in this study. (b) Cooling curves monitored in the $0.2 \mathrm{C}$ sample at 10 and $15 \mathrm{~mm}$ heights from the copper mold. 


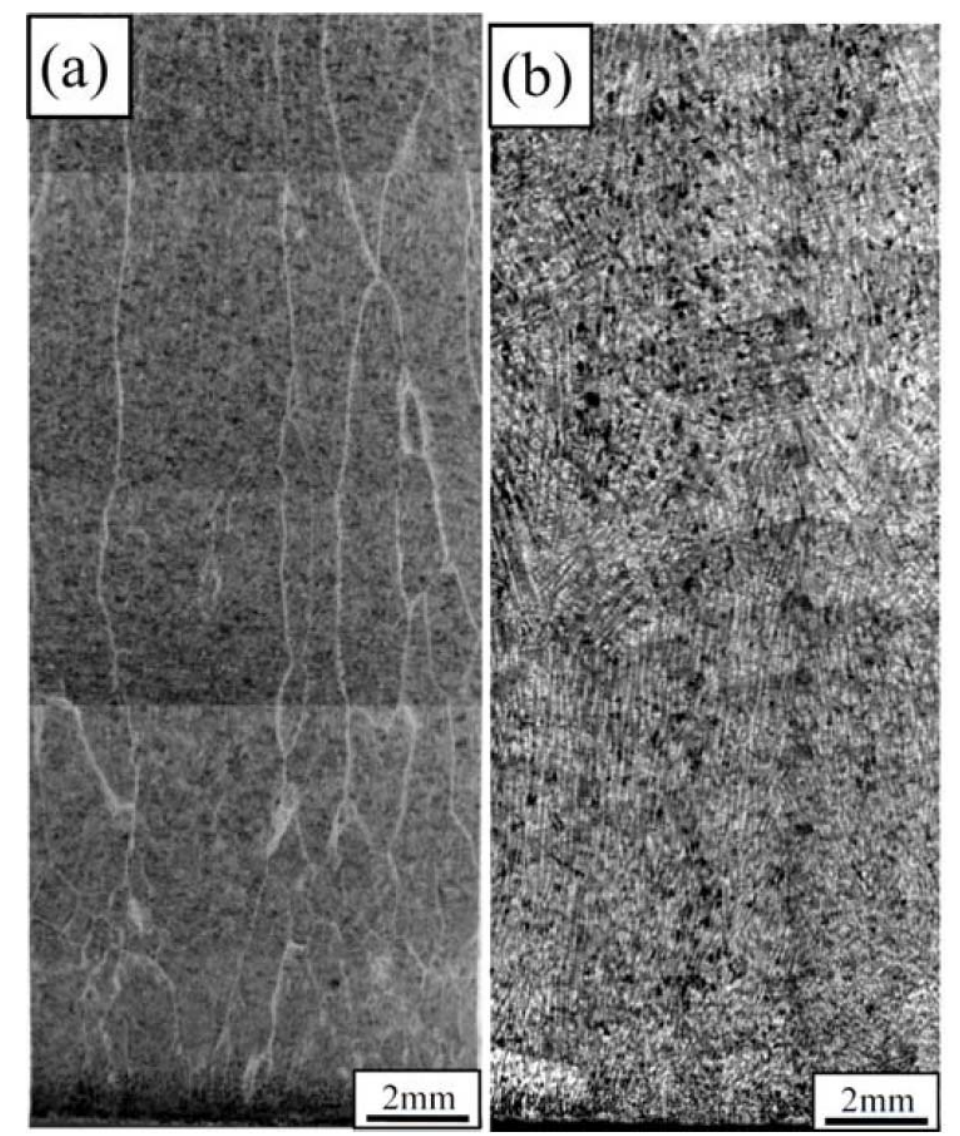

Fig. 3. Micrographs of the $0.2 \mathrm{C}$ sample naturally cooled to RT showing (a) as-cast $\gamma$

structure and (b) dendrite structure. The bottom side and the upper side correspond to the mold side and the upper side of the ingot, respectively. The bright boundaries in Fig. 3(a) represent the as-cast $\gamma$ grain boundaries. These boundaries are visible because of the primary ferrite phase precipitating on the $\gamma$ grain boundary. 


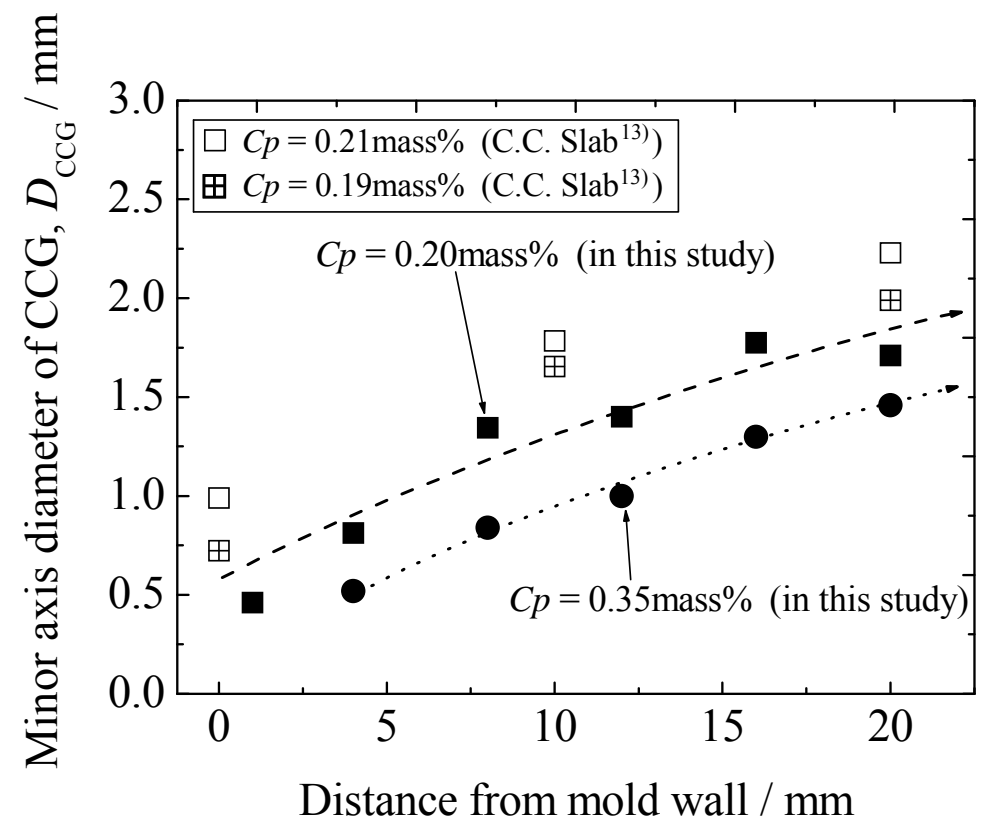

Fig. 4. Relationship between the minor axis diameter, $D_{\mathrm{CCG}}$, and the distance from mold wall in $0.2 \mathrm{C}$ and $0.35 \mathrm{C}$ samples. Also, the corresponding data for the C.C. slab [13] are plotted for comparison. 

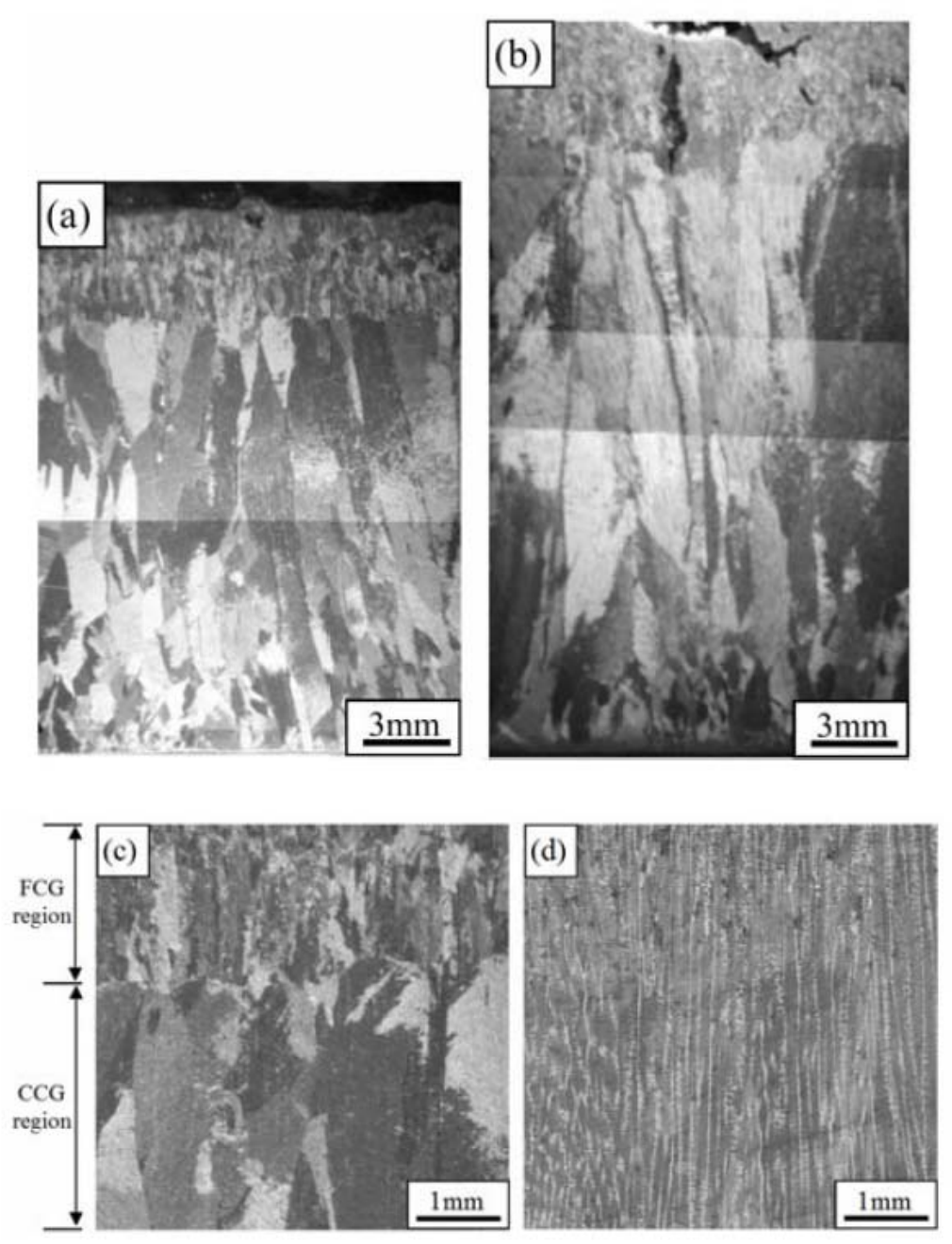

Fig. 5. Micrographs of the structures in $0.2 \mathrm{C}$ sample quenched at different times. As-cast $\gamma$ structure in $0.2 \mathrm{C}$ sample quenched at (a)30s, (b)50s. (c) Enlargement of the FCG/CCG region boundary in $0.2 \mathrm{C}$ sample quenched at $30 \mathrm{~s}$. (d) Dendrite structure in the same region as in Fig. 5(c). The bottom side and the upper side correspond to the mold side and the upper side of the ingot, respectively. 


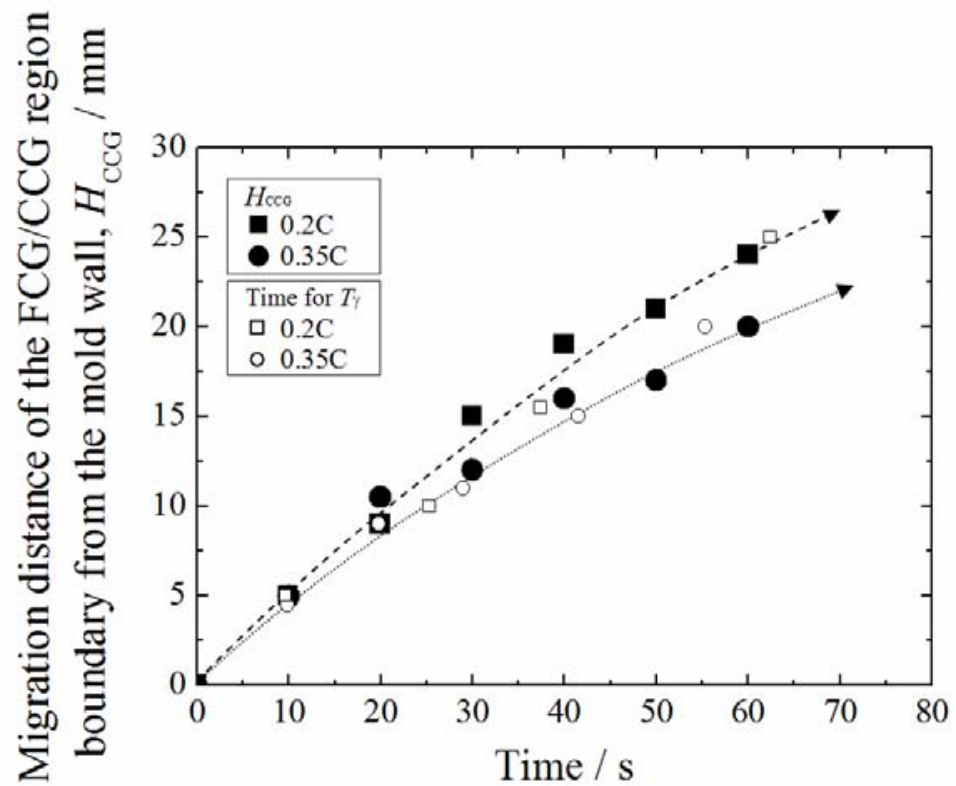

Fig. 6. Relationship between $H_{\mathrm{CCG}}$ and the holding time in $0.2 \mathrm{C}$ and $0.35 \mathrm{C}$ samples. The open marks indicate the time when the temperature reaches $T_{\gamma}$ in the cooling curve at each position. 


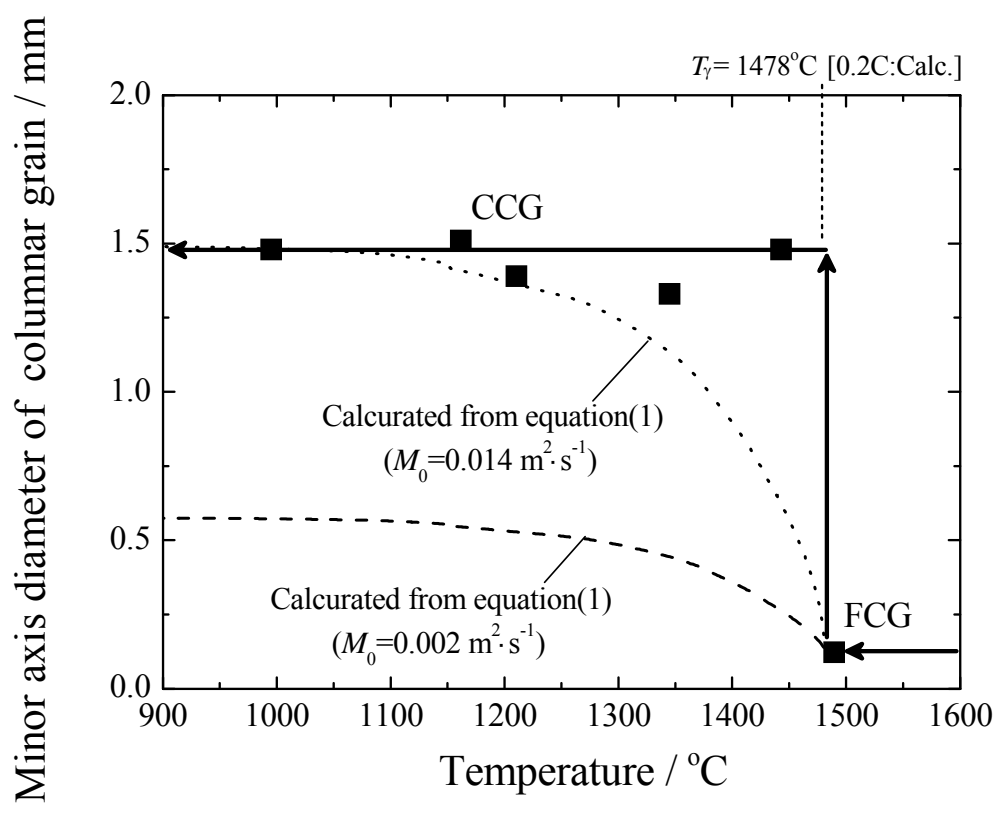

Fig. 7. Comparison of the $\gamma$ grain growth behavior at a position in $0.2 \mathrm{C}$ steel $8 \mathrm{~mm}$ away from mold with those calculated from the equation (1). 


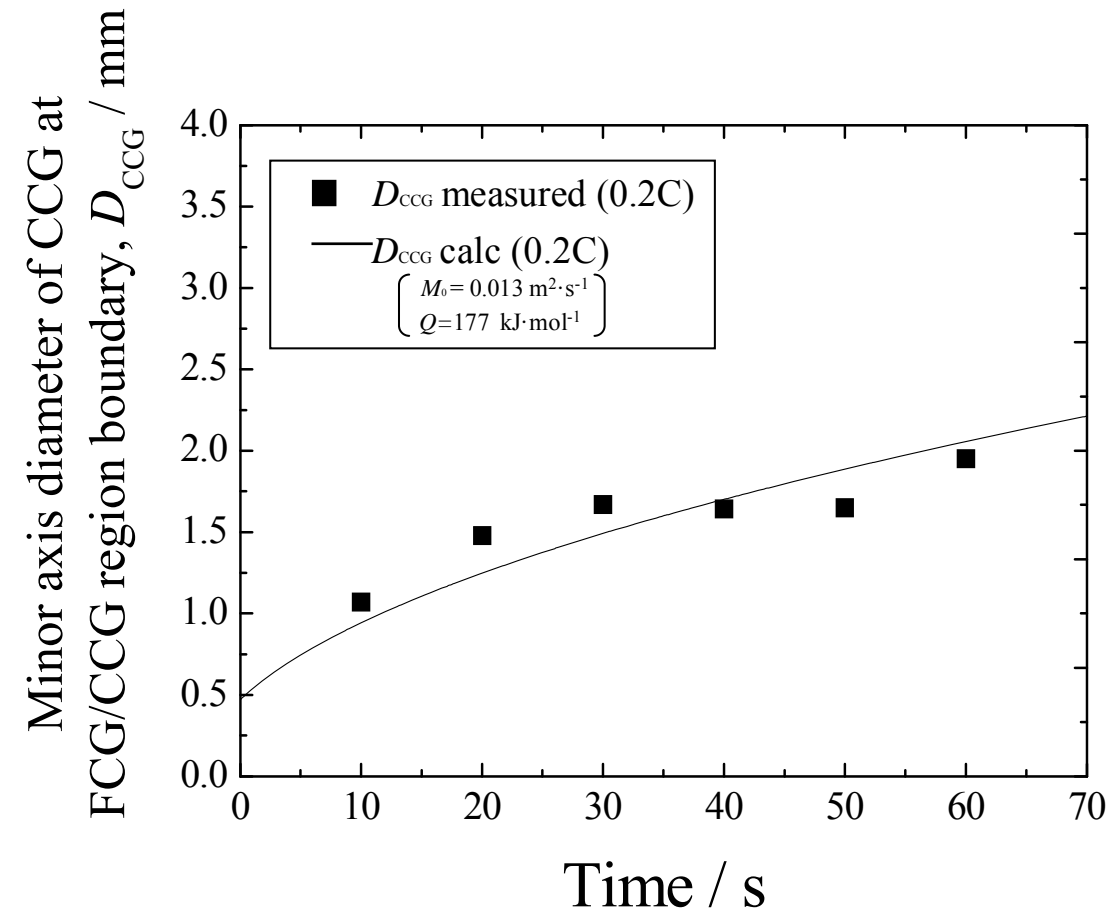

Fig. 8. Relationship between $D_{\mathrm{CCG}}$ at the FCG/CCG region boundary and the time after casting. Solid line was calculated from equation (1). 
(a)

(i)

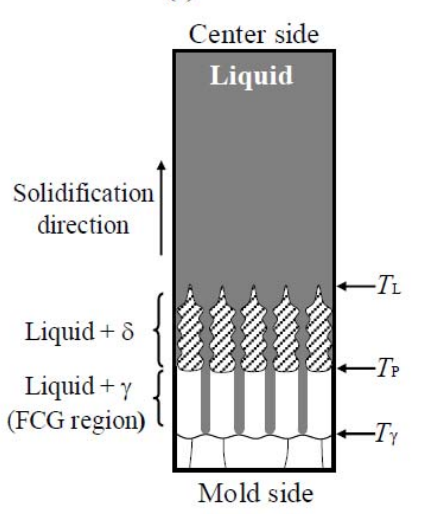

(ii)

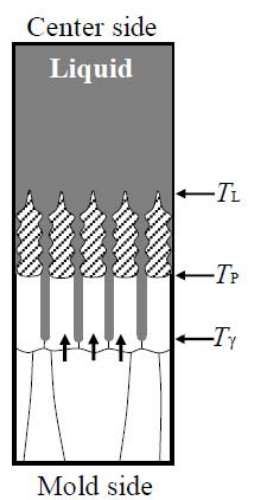

(b)

(iii)

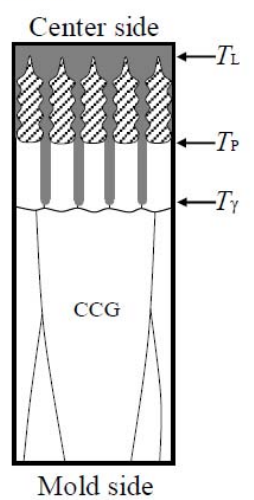

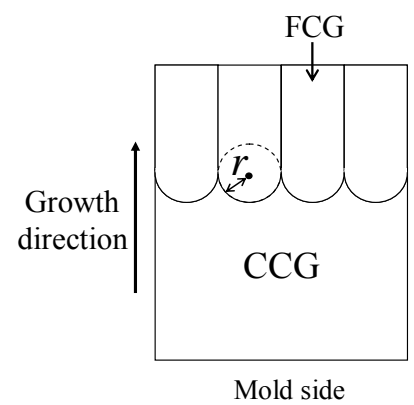

Mold side

$T_{\mathrm{L}}$ Liquidus temperature

$T_{\mathrm{P}}$ Peritectic temperature

$T_{\gamma}$ Temperature for completion of $\gamma$ transformation

Fig. 9. (a) Schematic illustration of the formation process of the CCG structure

observed in the present study. The solidification proceeds from (i) to (iii). (b)

Schematic illustration for the migration of FCG/CCG region boundary. 